\title{
Hemograma e proteinograma de cutias (Dasyprocta sp.) hígidas, criadas em cativeiro: influência do sexo e da idade.
}

[Haemogram and proteinogram of healthy agouti (Dasyprocta sp.) raised in captivity: influence of gender and age]

\author{
E.E.A. Ribeiro ${ }^{1}$, M.C.S. Batista ${ }^{2}$, M.A.M. Carvalho ${ }^{2}$, J.A.L. Silva ${ }^{3}$ \\ ${ }^{1}$ Aluno de pós-graduação - CCA-UFPI - Teresina, PI \\ ${ }^{2}$ Departamento de Morfofisiologia Veterinária - CCA-UFPI \\ Campus da Socopo \\ 64049-550 - Teresina-PI \\ ${ }^{3}$ Departamento de Planejamento e Políticas Agrícolas - CCA-UFPI - Teresina, PI
}

\begin{abstract}
RESUMO
Determinaram-se os perfis do hemograma e do proteinograma de 24 cutias (Dasyprocta sp.) saudáveis, criadas em cativeiro, e avaliaram-se a influência de sexo e de idade e a interação sexo-idade. $O$ delineamento foi inteiramente ao acaso em arranjo fatorial $2 \times 4$ (dois sexos e quatro faixas etárias), com três repetições. Os valores do hemograma e do proteinograma foram: leucócitos totais: 7,24 $\pm 2,52$ $\left(\mathrm{x} 10^{3} / \mu \mathrm{l}\right)$, basófilos: $0,11 \pm 0,2\left(\mathrm{x} 10^{3} / \mu \mathrm{l}\right)$, eosinófilos: $0,32 \pm 0,2\left(\mathrm{x} 10^{3} / \mu \mathrm{l}\right)$, segmentados: $2,5 \pm 1,22\left(\mathrm{x} 10^{3} / \mu \mathrm{l}\right)$,

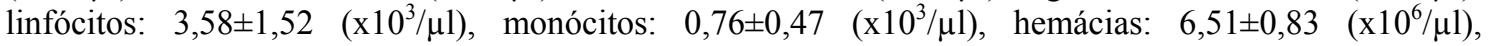
hematócrito: 43,58 $\pm 3,41 \%$, hemoglobina: $12,71 \pm 1,04 \mathrm{~g} / \mathrm{dl}$, volume corpuscular médio: $67,61 \pm 7,52 \mu \mathrm{l}$, concentração de hemoglobina corpuscular média: $29,19 \pm 1,61 \%$, plaquetas: $150,08 \pm 63,39\left(\mathrm{x} 10^{3} / \mu 1\right)$, proteínas totais: $6,04 \pm 1,77 \mathrm{~g} / \mathrm{dl}$, albumina: $2,07 \pm 0,79 \mathrm{~g} / \mathrm{dl}$ e globulinas: $3,97 \pm 1,54 \mathrm{~g} / \mathrm{dl}$. Os parâmetros relativos à série vermelha e ao proteinograma não diferiram quanto ao sexo ou idade, e não houve interação sexo-idade. Os valores de linfócitos foram mais baixos entre os animais mais velhos.
\end{abstract}

Palavras-chave: cutia, Dasyprocta sp., hemograma, proteínas

\begin{abstract}
The profiles of hemogram and proteinogram of healthy agouti (Dasyprocta sp.) grown in captivity, in Piaui State, Brazil, were determined, as well as the influence of gender and age factors and the relationship between sex-age them. A completely randomized design was used in a factorial arrange $2 \times 4$, two genders and four ages, totaling 24 animals. The mean values of the hemogram were: erythrocytes $=$ $6.51 \pm 0.83\left(\times 10^{6}\right)$; hematocrit $=43.58 \pm 3,41 \%$; hemoglobin $=12.71 \pm 1,04 \mathrm{~g} / \mathrm{dl} ;$; mean corpuscular volume $=64.61 \pm 7.52 \mu \mathrm{l} ;$; mean corpuscular hemoglobin concentration $=29.19 \pm 1,61 \%$; platelets $=150.08 \pm 63.39$ $\left(x 10^{3} / \mu l\right) ;$ white blood cells $=7.24 \pm 2.52\left(\times 10^{3} / \mu l\right) ;$ basophils $=0.11 \pm 0,2\left(x 10^{3} / \mu l\right) ;$ eosinophils $=$ $0,32 \pm 0,2\left(x 10^{3} / \mu l\right) ;$ neutrophils $=2.5 \pm 1,22\left(x 10^{3} / \mu l\right) ;$ lymphocytes $=3.58 \pm 1.52\left(x 10^{3} / \mu l\right)$; and monocytes $=0.76 \pm 0.47\left(x 10^{3} / \mu l\right)$. The mean values of the proteinogram were: total serum proteins $=6.04 \pm 1.77 \mathrm{~g} / \mathrm{dl}$; albumin $=2.07 \pm 0.79 \mathrm{~g} / \mathrm{dl}$ and globulin $=3.97 \pm 1.54 \mathrm{~g} / \mathrm{dl}$. Neither gender nor age influenced the results of the studied parameters. Association between gender and age was not verified. The values of lymphocytes were lower in older animals.
\end{abstract}

Keywords: agouti, Dasyprocta sp., hemogram, protein

\section{INTRODUÇ̃̃O}

A criação de cutias em cativeiro é uma das formas de preservação e exploração comercial, por servirem como fonte de proteína e outros insumos (Hosken, 2001). Estudos hematológicos de cutias já foram realizados por alguns autores (Pachaly, 1994; Mangrich et al., 1996; Amaro e

Recebido em 24 de julho de 2006

Aceito em 2 de setembro de 2008

E-mail: egledson@hotmail.com 
Sousa, 1996; Queiroz et al., 1996; Mangrich, 2000; Schmidt-Popazoglo et al., 2002ab).

Queiroz et al. (1996) encontram para machos e fêmeas (Dasyprocta primnolopha), respectivamente, leucócitos totais: 3,0-11,0/3,8$9,3 \times 10^{3} / \mu 1$; basófilos: $0 / 0 \%$; eosinófilos: $2-8 / 2$ 11\%; bastonetes: 0-5/0-3\%; segmentados: $56-$ 70/25-82\%; linfócitos: 19-36/10-58\%; monócitos: 1-4/1-7\%; hemácias: 4,7-6,4/4,7$5,8 \times 10^{6} / \mu 1$; hematócrito: 42-59/42-53\%; hemoglobina: $\quad 11,52-20,42 / 12,31-16,1 \mathrm{mg} / \mathrm{dl}$; volume corpuscular médio: 89,4-92,2/89,4$91,4 \mu 1$; concentração de hemoglobina corpuscular média:27,4-34,6/28,2-38,3\%.

Mangrich et al. (2000), ao avaliarem a influência do sexo e da idade em cutias (Dasyprocta azarae Lichenstein) jovens ( $\leq 8$ meses) e adultas $(>8$ meses), encontraram diferenças em relação ao número de hemácias, com valores de 7,23+0,18 $\times 10^{6} / \mu 1$ e de $7,91 \pm 0,26 \times 10^{6} / \mu 1$, para animais jovens e adultos, respectivamente, e concentração de proteínas totais de $6,11 \pm 0,11 \mathrm{~g} / \mathrm{dl}$.

Amaro e Sousa (1996), em cutias (Dasyprocta primnolopha), encontram valores de $4,43 \pm 0,77 \mathrm{~g} / \mathrm{dl}$ para proteínas totais e de $3,84 \pm 0,32 \mathrm{~g} / \mathrm{dl}$ para albumina.

Destaca-se que não foram encontrados, na literatura consultada, valores referentes a contagens de plaquetas.

Variações locais (clima e altitude), tipos raciais, o sexo e a idade interferem nos valores hematológicos das espécies animais (GarciaNavarro e Pachaly, 1994; Matos e Matos, 1995; Swenson, 1996; Dias Junior et al., 2006).

Swenson (1996) afirma que a partir da concepção ocorre uma diminuição constante da porcentagem de água corporal nos mamíferos com aumento das concentrações de eritrócitos e outros elementos do sangue que se estabilizam quando o animal atinge a maturidade.

Para Birgel et al. (1982), com o avançar da idade, a medula óssea transforma-se de vermelha em amarela e, posteriormente, converte-se em cinzenta, na qual, as células mesenquimais perdem sua totipotencialidade. Esses autores afirmam também que nos animais jovens o sistema linfóide predomina sobre o sistema mielóide, e, com o avançar da idade, o sistema mielóide sobressai em relação ao linfóide.

Segundo Kerr (2003), os machos tendem a apresentar maiores valores para volume globular (HT), em razão de os hormônios masculinos serem estimuladores das células-tronco. Ferreira (2002), entretanto, atribuiu o maior valor para HT nos machos ao fato de os hormônios femininos inibirem à eritropoese.

Deste modo, para o melhor entendimento da espécie, análises hematológicas estratificadas em relação ao sexo e à idade se fazem necessárias para que se possa observar a influência desses fatores.

Este trabalho teve por objetivo determinar os perfis do hemograma e do proteinograma de cutias (Dasyprocta sp.) hígidas, criadas em cativeiro, de acordo com o sexo, a idade e a interação sexo-idade.

\section{MATERIAL E MÉTODO}

O estudo foi realizado no período de setembro de 2004 a janeiro de 2005, com base no trabalho de Ribeiro et al. (2008).

Para a realização dos hemogramas, adotou-se o método da contagem manual de células em câmara de Neubauer. As diluições destinadas à contagem da série vermelha e à leucometria global foram feitas com solução fisiológica (Matos e Matos, 1995) e líquido de Turk (Garcia-Navaro e Pachaly, 1994), respectivamente, e para a contagem de plaquetas empregou-se solução à base de citrato de sódio (Matos e Matos, 1995). As contagens diferenciais de leucócitos foram realizadas pela contagem de cem células leucocitárias nos esfregaços sangüíneos corados por panótico, segundo Matos e Matos (1995).

O volume globular ou hematócrito (HT) foi determinado pelo método do microematócrito (Birgel et al., 1982), e a hemoglobina por espectrofotometria com o uso de kit comercial ${ }^{1}$. Os índices eritrocitários absolutos, volume corpuscular médio (VCM) e concentração de hemoglobina corpuscular média (CHCM), foram definidos por fórmulas matemáticas (Matos e Matos, 1995). Os teores de proteínas totais e

${ }^{1}$ Labteste S.A. - Lagoa Santa, MG - Brasil. 
albumina foram determinados por espectrofotometria com o uso de kits comerciais.

Os resultados obtidos foram submetidos à análise de variância e ao teste Tukey $(\mathrm{P}<0,05)$. Utilizouse do pacote estatístico SAEG (Sistema..., 1983).

\section{RESULTADOS E DISCUSSÃO}

A temperatura retal média foi de $39,17 \pm 0,77^{\circ} \mathrm{C}$ e a massa corporal média foi de $2,15 \pm 0,34 \mathrm{~kg}$ (Ribeiro et al., 2008).

Os resultados para a série branca do sangue encontram-se na Tab. 1. Foram observados valores relativos menores para neutrófilos e maiores para linfócitos e monócitos, tanto para machos como para fêmeas, que os relatados por Queiroz et al. (1996). Isso talvez se deva ao número de animais abaixo de um ano de idade, cujo sistema linfóide é mais significativo do que o sistema mielóide (Birgel et al., 1982).

Os valores percentuais dos eosinófilos e dos segmentados aumentaram e os de linfócitos diminuíram com aumento da idade. Para os valores absolutos dos leucócitos, dentro das faixas etárias, somente os segmentados mostraram diferenças significativas (Tab. 1). Estas observações não foram feitas por Mangrich et al. (2000), mas estão de acordo com os resultados de Birgel et al. (1982).

Tabela 1. Valores do leucograma de cutias (Dasyprocta sp.) saudáveis, criadas em cativeiro

\begin{tabular}{|c|c|c|c|c|c|c|c|c|c|c|c|}
\hline \multirow{3}{*}{ Variáveis } & \multirow{3}{*}{$\begin{array}{c}\mathrm{LT} \\
\left(\mathrm{x} 10^{3} / \mu \mathrm{l}\right)\end{array}$} & \multicolumn{10}{|c|}{ Leucometria diferencial $\left(\mathrm{x} 10^{3} / \mathrm{ul}\right)$} \\
\hline & & \multicolumn{2}{|c|}{ BAS } & \multicolumn{2}{|c|}{ EOS } & \multicolumn{2}{|c|}{ SEG } & \multicolumn{2}{|c|}{ LIN } & \multicolumn{2}{|c|}{ MON } \\
\hline & & $\begin{array}{l}\mathrm{ABS} \\
\mathrm{n} \% / u 1\end{array}$ & $\begin{array}{r}\text { REL } \\
\%\end{array}$ & $\begin{array}{l}\mathrm{ABS} \\
\mathrm{n} \% / u 1\end{array}$ & $\begin{array}{r}\text { REL } \\
\%\end{array}$ & $\begin{array}{l}\mathrm{ABS} \\
\mathrm{n} \% / u 1\end{array}$ & $\begin{array}{r}\text { REL } \\
\%\end{array}$ & $\begin{array}{l}\mathrm{ABS} \\
\mathrm{n} \% / u \mathrm{l}\end{array}$ & $\begin{array}{r}\text { REL } \\
\%\end{array}$ & $\begin{array}{l}\mathrm{ABS} \\
\mathrm{n} / u 1\end{array}$ & $\begin{array}{c}\text { REL } \\
\%\end{array}$ \\
\hline Média geral & 7,24 & 0,11 & 1,17 & 0,32 & 4,63 & 2,5 & 33,71 & 3,58 & 50,29 & 0,76 & 10,21 \\
\hline Desvio & 2,52 & 0,20 & 1,24 & 0,20 & 2,93 & 1,22 & 11,3 & 1,52 & 14,4 & 0,47 & 4,40 \\
\hline $\begin{array}{l}\text { Dadivan } \\
\text { Machos }\end{array}$ & $7,42 \mathrm{a}$ & $0,06 \mathrm{a}$ & $0,92 \mathrm{a}$ & $0,29 \mathrm{a}$ & $3,92 \mathrm{a}$ & $2,67 a$ & $34,6 \mathrm{a}$ & $3,64 a$ & $50,8 \mathrm{a}$ & $0,76 \mathrm{a}$ & $9,75 \mathrm{a}$ \\
\hline Fêmeas & $7,05 \mathrm{a}$ & $0,16 \mathrm{a}$ & $1,42 \mathrm{a}$ & $0,36 \mathrm{a}$ & $5,33 \mathrm{a}$ & $2,32 \mathrm{a}$ & $32,8 \mathrm{a}$ & $3,53 \mathrm{a}$ & $49,8 \mathrm{a}$ & $0,76 \mathrm{a}$ & $10,7 \mathrm{a}$ \\
\hline Idade $<1$ ano & $6,65 \mathrm{a}$ & $0,09 \mathrm{a}$ & $1,5 \mathrm{a}$ & $0,16 \mathrm{a}$ & $2,0 \mathrm{~b}$ & $1,56 \mathrm{~b}$ & $23,2 b$ & $4,33 \mathrm{a}$ & $66,2 \mathrm{a}$ & $0,52 \mathrm{a}$ & $7,7 \mathrm{a}$ \\
\hline Idade $1 \leq x<2$ & $7,05 \mathrm{a}$ & $0,05 a$ & $1,0 \mathrm{a}$ & $0,34 a$ & $4,8 \mathrm{ab}$ & $2,6 a b$ & $35,3 \mathrm{ab}$ & $3,3 \mathrm{a}$ & $48,8 \mathrm{ab}$ & $0,72 \mathrm{a}$ & $10,0 \mathrm{a}$ \\
\hline Idade $2 \leq x<3$ & $8,87 \mathrm{a}$ & $0,21 \mathrm{a}$ & $0,67 \mathrm{a}$ & $0,39 a$ & $5,0 \mathrm{ab}$ & $3,4 \mathrm{a}$ & $38,5 \mathrm{a}$ & $4,01 \mathrm{a}$ & $44,5 \mathrm{ab}$ & $1,01 \mathrm{a}$ & $11,3 \mathrm{a}$ \\
\hline Idade $3 \leq x$ & $6,38 \mathrm{a}$ & $0,10 \mathrm{a}$ & $1,50 \mathrm{a}$ & $0,41 \mathrm{a}$ & $6,7 \mathrm{a}$ & $2,4 a b$ & $37,8 \mathrm{ab}$ & $2,70 \mathrm{a}$ & $41,7 \mathrm{~b}$ & $0,79 a$ & $12,3 \mathrm{a}$ \\
\hline Machos $<1$ & $5,37 \mathrm{a}$ & $0,09 a$ & $1,67 \mathrm{a}$ & $0,10 \mathrm{a}$ & $1,33 \mathrm{a}$ & $1,01 \mathrm{a}$ & $19,0 \mathrm{a}$ & $3,89 \mathrm{a}$ & $73,0 \mathrm{a}$ & $0,29 a$ & $5,00 \mathrm{a}$ \\
\hline Fềmeas $<1$ & $7,93 a$ & $0,09 \mathrm{a}$ & $1,33 \mathrm{a}$ & $0,22 \mathrm{a}$ & $2,67 \mathrm{a}$ & $2,11 \mathrm{a}$ & $27,3 \mathrm{a}$ & $4,76 \mathrm{a}$ & $59,3 \mathrm{a}$ & $0,75 \mathrm{a}$ & $9,33 \mathrm{a}$ \\
\hline $1 \leq$ Machos $<2$ & $9,09 a$ & $0,05 \mathrm{a}$ & $0,67 \mathrm{a}$ & $0,35 \mathrm{a}$ & $3,67 \mathrm{a}$ & $3,81 \mathrm{a}$ & $44,0 \mathrm{a}$ & $3,85 \mathrm{a}$ & $40,3 \mathrm{a}$ & $1,02 \mathrm{a}$ & $11,3 \mathrm{a}$ \\
\hline $1 \leq$ Fêmeas $<2$ & $5,01 \mathrm{a}$ & $0,05 a$ & $1,33 \mathrm{a}$ & $0,32 \mathrm{a}$ & $6,00 \mathrm{a}$ & $1,47 \mathrm{a}$ & $26,7 \mathrm{a}$ & $2,75 \mathrm{a}$ & $57,3 \mathrm{a}$ & $0,42 \mathrm{a}$ & $8,67 \mathrm{a}$ \\
\hline $2 \leq$ Machos $<3$ & $9,67 \mathrm{a}$ & $0,07 \mathrm{a}$ & $0,67 \mathrm{a}$ & $0,37 \mathrm{a}$ & $4,33 \mathrm{a}$ & $3,70 \mathrm{a}$ & 36,7 & $4,41 \mathrm{a}$ & $46,6 \mathrm{a}$ & $1,12 \mathrm{a}$ & $11,7 \mathrm{a}$ \\
\hline $2 \leq$ Fêmeas $<3$ & $8,07 \mathrm{a}$ & $0,34 \mathrm{a}$ & $0,67 \mathrm{a}$ & $0,41 \mathrm{a}$ & $5,67 \mathrm{a}$ & $3,10 \mathrm{a}$ & $40,3 \mathrm{a}$ & $3,61 \mathrm{a}$ & $42,3 \mathrm{a}$ & $0,90 \mathrm{a}$ & $11,0 \mathrm{a}$ \\
\hline Machos $\geq 3$ & $7,19 a$ & $0,16 a$ & $2,33 a$ & $0,48 \mathrm{a}$ & $7,00 \mathrm{a}$ & $2,61 \mathrm{a}$ & $37,0 \mathrm{a}$ & $2,99 \mathrm{a}$ & $40,0 \mathrm{a}$ & $0,96 \mathrm{a}$ & $13,7 \mathrm{a}$ \\
\hline Fêmeas $\geq 3$ & $5,57 \mathrm{a}$ & $0,04 \mathrm{a}$ & $0,67 \mathrm{a}$ & $0,35 \mathrm{a}$ & $6,33 \mathrm{a}$ & $2,16 \mathrm{a}$ & $38,7 \mathrm{a}$ & $2,41 \mathrm{a}$ & $43,3 \mathrm{a}$ & $0,61 \mathrm{a}$ & $11,0 \mathrm{a}$ \\
\hline
\end{tabular}

LT: leucócitos totais, BAS: basófilos; EOS: eosinófilos; LIN: linfócitos; MON: monócitos, ABS: valor absoluto, REL: valor relativo.

.Letras diferentes na mesma coluna indicam diferença significativa $(\mathrm{P}<0,05$.

Os resultados da série vermelha encontram-se na Tab. 2. Verifica-se, em relação aos achados de Queiroz et al. (1996), contagem maior para as hemácias e valores menores para o hematócrito e a hemoglobina. Ressalta-se que a maior ou menor disponibilidade de água nas baias, a idade dos animais, a habilidade do profissional na contagem de hemácias e até mesmo o tipo de solução diluidora podem interferir nas concentrações das variáveis da série vermelha. Os resultados são controversos, sem levar em conta sexo e idade. Quanto aos outros parâmetros da série vermelha não foram observadas diferenças significativas com outros trabalhos (Pachaly, 1994; Mangrich et al., 1996; Amaro e 
Sousa, 1996; Queiroz et al., 1996; Mangrich, 2000; Schmidt-Popazoglo et al., 2002ab).

Os parâmetros relativos à série vermelha e ao proteinograma não diferiram significativamente em relação ao sexo ou à idade, e não houve interação sexo-idade. Para as hemácias, o resultado difere do encontrado por Mangrich (2000), que verificou maior número de hemácias em cutias adultas.

As diferenças no número de plaquetas, nas idades estudadas, não foram significativas.

Tabela 2. Valores do eritrograma, plaquetas e proteinograma de cutias (Dasyprocta sp.) saudáveis, criadas em cativeiro

\begin{tabular}{|c|c|c|c|c|c|c|c|c|c|}
\hline Variável & $\begin{array}{c}\text { HM } \\
(\mathrm{x} 106 / \mathrm{u})\end{array}$ & $\begin{array}{l}\mathrm{HT} \\
(\%)\end{array}$ & $\begin{array}{c}\mathrm{HB} \\
(\mathrm{g} / \mathrm{dl})\end{array}$ & $\begin{array}{c}\mathrm{VCM} \\
\text { (fl) }\end{array}$ & $\begin{array}{c}\text { CHCM } \\
(\%)\end{array}$ & $\begin{array}{c}\text { PLT } \\
\left(\mathrm{x} 10^{3} / \mathrm{ul}\right) \\
\end{array}$ & $\begin{array}{c}\text { PT } \\
(\mathrm{g} / \mathrm{dl})\end{array}$ & $\begin{array}{l}\text { ALB } \\
(\mathrm{g} / \mathrm{dl})\end{array}$ & $\begin{array}{c}\mathrm{GB} \\
(\mathrm{g} / \mathrm{dl})\end{array}$ \\
\hline Média geral & 6,51 & 43,53 & 12,71 & 67,61 & 29,19 & 150,8 & 6,04 & 2,07 & 3,97 \\
\hline Desvio Padrão & 0,83 & 3,41 & 1,04 & 7,52 & 1,61 & 63,39 & 1,77 & 0,79 & 1,54 \\
\hline Machos & $6,69 a$ & $44,08 \mathrm{a}$ & $12,87 \mathrm{a}$ & $66,35 \mathrm{a}$ & $29,26 \mathrm{a}$ & $143,2 \mathrm{a}$ & $6,27 \mathrm{a}$ & $2,31 \mathrm{a}$ & $3,95 \mathrm{a}$ \\
\hline Fêmeas & $6,34 a$ & $43,08 \mathrm{a}$ & $12,55 \mathrm{a}$ & $68,87 \mathrm{a}$ & $29,11 \mathrm{a}$ & $157,0 \mathrm{a}$ & $5,81 \mathrm{a}$ & $1,83 \mathrm{a}$ & $3,98 \mathrm{a}$ \\
\hline Idade $<1$ ano & $6,29 a$ & $44,83 \mathrm{a}$ & $12,93 \mathrm{a}$ & $72,77 \mathrm{a}$ & $28,81 \mathrm{a}$ & $142,5 \mathrm{a}$ & $5,48 \mathrm{a}$ & $2,02 \mathrm{a}$ & $3,46 \mathrm{a}$ \\
\hline Idade $1 \leq 2$ & $6,42 \mathrm{a}$ & $43,5 \mathrm{a}$ & $13,06 \mathrm{a}$ & $68,02 \mathrm{a}$ & $30,03 a$ & $125,3 \mathrm{a}$ & $6,83 \mathrm{a}$ & $2,32 \mathrm{a}$ & $4,52 \mathrm{a}$ \\
\hline Idade $2 \leq 3$ & $6,85 \mathrm{a}$ & $44,0 \mathrm{a}$ & $12,73 \mathrm{a}$ & $64,72 \mathrm{a}$ & $29,07 \mathrm{a}$ & $163,3 \mathrm{a}$ & $4,9 a$ & $1,87 \mathrm{a}$ & $3,03 \mathrm{a}$ \\
\hline Idade $>3$ & $6,5 \mathrm{a}$ & $42,0 \mathrm{a}$ & $12,11 \mathrm{a}$ & $64,95 \mathrm{a}$ & $28,82 \mathrm{a}$ & $169,3 \mathrm{a}$ & $6,94 a$ & $2,08 \mathrm{a}$ & $4,86 a$ \\
\hline Machos $<1$ ano & $6,56 a$ & $44,00 \mathrm{a}$ & $12,24 \mathrm{a}$ & $67,94 a$ & $27,81 \mathrm{a}$ & $93,00 \mathrm{a}$ & $4,67 \mathrm{a}$ & $1,7 \mathrm{a}$ & $2,97 \mathrm{a}$ \\
\hline Fêmeas $<1$ ano & $6,02 \mathrm{a}$ & $45,67 \mathrm{a}$ & $13,62 \mathrm{a}$ & $77,60 \mathrm{a}$ & $29,82 \mathrm{a}$ & $191,7 \mathrm{a}$ & $6,30 \mathrm{a}$ & $2,35 \mathrm{a}$ & $3,95 \mathrm{a}$ \\
\hline Machos $1 \leq 2$ & $6,04 a$ & $42,67 \mathrm{a}$ & $13,20 \mathrm{a}$ & $70,70 \mathrm{a}$ & $30,88 \mathrm{a}$ & $104,7 \mathrm{a}$ & $7,58 \mathrm{a}$ & $2,52 \mathrm{a}$ & $5,06 \mathrm{a}$ \\
\hline Fêmeas $1 \leq 2$ & $6,81 \mathrm{a}$ & $44,33 \mathrm{a}$ & $12,92 \mathrm{a}$ & $65,33 \mathrm{a}$ & $29,18 \mathrm{a}$ & $146,0 \mathrm{a}$ & $6,08 \mathrm{a}$ & $2,11 \mathrm{a}$ & $3,97 \mathrm{a}$ \\
\hline Machos $2 \leq 3$ & $7,49 a$ & $46,67 \mathrm{a}$ & $13,46 \mathrm{a}$ & $62,43 \mathrm{a}$ & $29,12 \mathrm{a}$ & $180,0 \mathrm{a}$ & $5,69 a$ & $2,71 \mathrm{a}$ & $2,98 \mathrm{a}$ \\
\hline Fêmeas $2 \leq 3$ & $6,20 \mathrm{a}$ & $41,33 \mathrm{a}$ & $11,99 a$ & $67,00 \mathrm{a}$ & $29,02 \mathrm{a}$ & $146,7 \mathrm{a}$ & $4,11 \mathrm{a}$ & $1,04 a$ & $3,08 \mathrm{a}$ \\
\hline Machos $>3$ & $6,68 \mathrm{a}$ & $43,00 \mathrm{a}$ & $12,57 \mathrm{a}$ & $64,33 a$ & $29,23 \mathrm{a}$ & $195,0 \mathrm{a}$ & $7,13 a$ & $2,33 a$ & $4,81 \mathrm{a}$ \\
\hline Fêmeas $>3$. & $6,32 \mathrm{a}$ & $41,00 \mathrm{a}$ & $11,66 \mathrm{a}$ & $65,56 \mathrm{a}$ & $28,42 \mathrm{a}$ & $143,7 \mathrm{a}$ & $6,74 a$ & $1,83 \mathrm{a}$ & $4,91 \mathrm{a}$ \\
\hline
\end{tabular}

HM: hemácias, HB: hemoglobina, HT: hematócrito, VCM: volume corpuscular médio, CHCM: concentração de hemoglobina corpuscular média, PLT: plaquetas, PT: proteínas totais, ALB: albumina, GB: globulina.

Letras diferentes na mesma coluna, indicam diferença significativa $(\mathrm{P}<0,05)$.

Para as proteínas totais, os valores encontrados foram semelhantes aos observados por Mangrich (2000) e maiores que os citados por Amaro e Sousa (1996). O grau de hidratação, a idade dos animais, o nível de estimulação imunológica e a dieta são fatores que influenciam na mensuração desta variável.

As concentrações de albumina foram bem mais baixas que as verificadas por Amaro e Sousa (1996). Provavelmente, a dieta dos animais em estudo seja a explicação mais adequada para essa diferença.

Em relação às globulinas, entre os autores consultados, não foram encontradas citações sobre seus níveis, nos animais pesquisados.
Os resultados deste trabalho podem servir de referência para este grupo de animais e mostram a necessidade de pesquisas hematológicas que levem em conta o sexo, a idade, o número maior de amostras e o maior espaço de tempo para que se possa observar a influência das estações do ano nas respostas do hemograma e do proteinograma.

\section{REFERÊNCIAS BIBLIOGRÁFICAS}

AMARO, K.M.; SOUZA, M.S.N. Determinação de proteínas e frações séricas em cutias (Dasyprocta primnolopha) mantidas em cativeiro. In: CONGRESSO PANAMERICANO DE CIÊNCIA VETERINÁRIA, 15., 1996, 
Campo Grande. Anais.... Campo Grande, 1996, p.74. (Resumo).

BIRGEL， E.H.; LARSSON, M.H.M.A.; HAGIWARA, M.K. et al. Patologia clínica veterinária. São Paulo: SPMV, 1982. 260p.

DIAS JUNIOR, R.F.; BRACARENSE, A.P.F.R.L.; MARÇAL, W.S. et al. Valores de referência e influência da idade no eritrograma de fêmeas bovinas da raça Aquitânica. Arq. Bras. Med. Vet. Zootec., v.58, p.311-315, 2006.

FERREIRA, A.F. Valores de referência do eritrograma e teores plasmáticos da proteína total e fibrinogênio de ovinos (Ovis aries, Linnaeus, 1758) da raça Santa Inês, criados na mesorregião metropolitana de Recife. Influência dos fatores sexual e etário. 2002. 33f. Dissertação (Mestrado) - Escola de Veterinária Universidade Federal Rural Pernambuco, Recife.

GARCIA-NAVARRO, C.E.; PACHALY, J.R. (Eds). Manual de hematologia veterinária. São Paulo: Varela, 1994. 169p.

HOSKEN, F.M. (Ed). Criação de cutias. Viçosa: Aprenda Fácil, 2001. 234p.

KERR, M.G. (Ed.). Exames laboratoriais em medicina veterinária: Bioquímica clínica e hematologia. São Paulo: Roca, 2003, 436p.

MANGRICH, R.M.V. Contribuição ao estudo dos valores normais de hemograma de cutias (Dasyprocta azarae Lichenstein). 2000. 60f. Dissertação (Mestrado) - Escola de Veterinária, Universidade Federal do Paraná, Curitiba.

MANGRICH， R.M.V.; PACHALY， J.R.; LANGE, R.R et al. Avaliação dos valores de hemograma de cutia (Dasyprocta agouti). In: CONGRESSO PANAMERICANO DE CIÊNCIA VETERINÁRIA, 15., 1996, Campo Grande. Anais... Campo Grande, 1996. p.70. (Resumo).

MATOS, M.S.; MATOS, P.F. Hematologia clínica. In: MATOS, M.S., MATOS, P.F. (Eds.).
Laboratório clínico médico veterinário. São Paulo: Atheneu, 1995. 320p.

PACHALY, J.R. Hematologia dos animais selvagens. In: KANTEK GARCIA-NAVARRO, C.E.; PACHALY, J.R. (Eds). Manual de hematologia veterinária. São Paulo: Varela, 1994. 174p.

QUEIROZ, P.V.S.; REIS, R.K.; GOLDBARG, M. et al. Aspectos hematológicos das cutias (Dasyprocta primnolopha) da região do semiárido nordestino. In: CONGRESSO PANAMERICANO CIÊNCIA VETERINÁRIA, 15., 1996, Campo Grande. Anais... Campo Grande, 1996, p.67. (Resumo).

RIBEIRO, E.E.A.; BATISTA, M.C.S.; CARVALHO, M.A.M et al. Dosagens iônicas e enzimáticas de cutias (Dasyprocta sp.) hígidas, criadas em cativeiro: Influência do sexo e da idade. Arq. Bras. Med. Vet. Zootec, v.60, p.651655, 2008.

SCHMIDT-POPAZOGLO, E.; LANGE，R.R.; PACHALY, J.R. et al. Determinação de parâmetros de bioquímica plasmática de cutias (Dasyprocta leporina) em cativeiro. In: CONGRESSO BRASILEIRO DE ZOOLOGIA, 24., 2002, Itajaí. Anais... Itajaí, 2002a, p.535. (Resumo).

SCHMIDT-POPAZOGLO, E.; LANGE, R.R.; PACHALY, J.R. et al. Determinação de parâmetros do hemograma de cutias (Dasyprocta leporina) em cativeiro. In: CONGRESSO BRASILEIRO DE ZOOLOGIA, 24., 2002a, Itajaí. Anais... Itajaí, 2002b, p.535. (Resumo).

SWENSON, M.J. Propriedades fisiológicas e constituintes químicos e celulares do sangue. In: SWENSON, M.J.; REECE, W.O. (Eds). Dukes. Fisiologia dos animais domésticos. Rio de Janeiro: Guanabara Koogan, 1996. 856p.

SISTEMA de análises estatísticas - SAEG. Viçosa: UFV, 1983. 59p. 